\title{
USING SITUATION AWARENESS AS A MEASURE OF DRIVER HAZARD PERCEPTION ABILITY
}

Atefeh Katrahmani, Nima Ahmadi, Matthew Romoser

Industrial Engineering \& Engineering Management, Western New England University

Springfield, Massachusetts, USA

Email: Atefeh.katrahmani@wne.edu,Nima.ahmadi@wne.edu; Matthew.romoser@wne.edu

Summary: The present study investigated the effectiveness of a tablet-based hazard anticipation training program on teenage drivers. Verbal and eye tracking protocols were mapped to Endsley's three level model of situation awareness (SA) as a means of measuring schema development. Participants were trained with a tablet based training program containing hazard identification scenarios. After six months they were asked to drive a simulator and on-road drive with various hazard scenarios. Results showed a significant difference between trained teen drivers and placebo teen drivers, both in eye tracking and verbal protocol. Verbal protocol and eye tracking protocol of trained teen drivers showed higher order of situation awareness in either of Endsley's model levels. This means trained group were more capable of identifying and mitigating the hazards and verbalizing the future states of the environment. In conclusion, the tablet based hazard identification and anticipation training program could be an effective post-licensure training program to give better insight of "what is going on" in driving environment.

\section{INTRODUCTION}

Due to the higher crash rate of teens, several studies have been performed to identify the salient factors that trigger novice driving errors. Studies indicate that in crashes amongst groups of drivers, Hazard Perception (HP) errors are common (McKnight, A. J., \& McKnight, A. S. 2003, Braitman et al. 2008, Curry et al. 2011, McDonald et al. 2014). HP is defined as situation awareness to hazardous events. In the young-novice accidents, poor HP is the main reason (Mayhew, 2007). According to Sagberg and Bjørnskau (2006), HP has two separable components: the first one is the extent or degree that a hazard is perceived; the second one is the Response Time (RT) to a hazard. The lower the RT, the fewer crashes the driver involve with. Driving skill as well as said HP components are correlated with traffic crashes. Driving skills are categorized into two groups: basic driving skills (such as operating a vehicle and learning traffic rules) and cognitive skills (some of which are hazard identification and hazard mitigation). Each of which has a different learning curve. Teens can be proficient in basic driving skills quickly, even before the start of the supervised driving period (Goodwin, Foss, Margolis, \& Waller, 2010; Hall \& West, 1996). Meanwhile, higher order skills such as HP have a sharper learning curve. Teen drivers require much more time to develop associated mental patterns or schemas to master them.

Endsley's model of situation awareness can facilitate the assessment of hazard perception (HP) skills. Furthermore, it allows researchers to perform quantitative analysis of situation awareness. Endsley's model of situation awareness is composed of three distinct levels: events perception, events comprehension, and events prediction. In the context of driving, to achieve a high level of situation awareness, at level one, a driver has to look at various elements while driving. In this context, elements are traffic components or situation. Level two is understanding the concurrent 
situation based on the observed objects. Finally, at level three, the driver must successfully project the future behavior of components in the environment (Endsley, 1995).

Evaluation of a drivers' situation awareness according to Endsley's model is a challenge. Amongst the methods for mapping behaviors to Endsley's three levels, commentary driving as a non-intrusive approach is the most common one. Participants are asked to report their thoughts concurrently and constantly while driving. This process is beneficial to assess a driver's HP proficiency. Differences are observed between young-inexperienced and experienced drivers in regards to said components of HP. RT of an experienced driver to a given hazard, as one HP component, is less relative to a novice driver. A young-inexperienced driver may not even perceive a potential event as a hazardous situation to react to it, namely encountering latent hazards. The result indicated that there is no significant difference in RT amongst groups of drivers once facing actual hazards. Whereas, in case of encountering latent events, a younginexperienced driver rarely identifies potential events as a hazardous occasion to react to (Sagberg, F., \& Bjørnskau T. 2006).

To address this issue and to evaluate teens' schema development, Katrahmani et al. (2016) applied commentary driving techniques to eye tracking protocols in order to assess situation awareness, HP, and schema development of young novice drivers. DiMaggio (1997) defined schemas as organized patterns of actions and thoughts. Based on schemas, perceived information is organized and data relationship is defined. The level of situation awareness is related to the quality of schema. A driver with a well-developed schema can achieve a full level of situation awareness in a highly dynamic, challenging environment. In addition, processing time of traffic scenes is related to the schema development. Over time, cues that are indicative of a given situation are consolidated as a schema. The more exposure to various situations and cues increases, the quality of a schema increases. This allows for more efficient processing of objects by converting declarative knowledge to procedural knowledge of an event. Information is stored as a procedure or rule when a skill is learned for the first time. Practicing a skill consolidates each step of a given multistep procedure into a continuous process. In terms of cognitive skill, experienced drivers have more developed schemas than novice drivers and it allows them to process driving information faster. Crundell and Underwood's (1998) research on drivers' eye movement under various cognitive workloads showed that in terms of information processing ability, experienced drivers require less time to process objects than novice drivers. The appraisal of verbal protocol of teen drivers with experienced drivers showed that teens' situation awareness barely exceeds level one of Endsley's model, which is object perception in a driving environment. Teens' verbal reports were namely about what was being performed and / or what was happening; whereas, the verbal protocol of experienced drivers revealed that a higher level of situation awareness was achieved and contained projections of what could have happened. Moreover, teen drivers processed fewer objects in the driving environment than experienced drivers (Katrahmani et al. 2016).

\section{METHODS}

The present paper reports the results on a study of the effectiveness of a tablet-based training program on novice teen driver performance. Performance was assessed by using a combination of eye tracking and verbal protocol analysis during use of driving simulator and field drive. 
Participants were brought to the Center for Advanced Training Research and Naturalistic Studies (ATRANS) at Western New England University in Springfield, MA.

The research also attempted to determine whether or not a training program would affect the verbal protocols of teens. Verbal protocol analysis was used to assess how teens processed hazard cues in the driving environment. Verbal protocols were mapped using Endsley's 3-level model of situation awareness. Drivers who are trained and have encountered a number of different types of driving hazards over and over again should have more highly developed hazard recognition schema. As such, they should be capable of articulating aspects of upcoming latent hazards, indicating a quick progression through all three levels of Endsley's model: perception of the elements of the hazard (level 1), comprehension of the hazard's priority (level 2), and projection of future events in the environment (level 3).

\section{Participants}

A total of thirty-two participants were recruited for this study. The teen driver cohort (16 drivers), the placebo teen driver cohort (16 drivers). All teenage drivers were between the ages of sixteen and eighteen and had completed the supervised driving period but were still in first year of their independent driving careers. Gender was equally balanced within each group. A driving school in southwestern Massachusetts was engaged to assist with the recruitment of participants.

\section{Apparatus}

Engaged Driving Training System (EDTS). EDTS is a tablet-based hazard anticipation training program. In this program there are eleven driving scenarios, each with a latent hazard.

Participants were asked to hold an iPad Air tablet horizontally with both hands. The tablet itself was the steering wheel. By rotating the tablet clockwise or counterclockwise they were able to move a virtual vehicle left or right. Participants could use their right thumb to accelerate and decelerate until the full stop via a slide bar at right side of the tablet. They were able to scan side to side by using a horizontal slide bar at left side of the screen (Figure 1). Participants were asked to identify hazard areas by pushing the pause button, then tapping at the hazard area. If they did it correctly, the area became green.

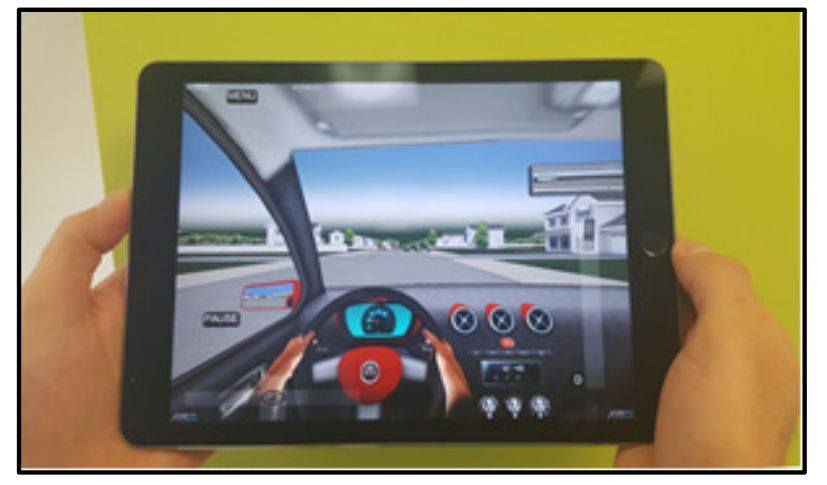

Figure 1. Engaged Driving Training System (EDTS) tablet

Driving Simulator. Scenarios were developed on the ATRANS STISIM V3 Mobile Driver Assessment \& Training Simulator (MODATS). MODATS is a portable 3-screen simulator. The simulator consists of a gaming chair and high-end pedal controls. Three 24" LCD monitors are mounted in front of the driver providing an approximately 150 degree field of view. 
Field Drive Vehicle. The field drive vehicle was a Toyota Prius belonging to the driving school who was responsible for recruiting the participants. Participants drove this car while the driving school's instructor was seated next to them and directing them.

Eye Tracker. Driver's roadway scanning was recorded using a Tobii Pro-II mobile eye tracker. The eye tracker consists of a lightweight pair of glasses with a scene camera and eye cameras integrated in to the frame. The driver's point of gaze, represented as a red circle, is superimposed upon the scene camera video image. The eye tracker used in this study is shown in Figure 3.

\section{Experimental Procedure}

The study took place in two sessions. The first session lasted approximately one hour. During this session, participants met with the study administrator in the Advanced Training Research and Naturalistic Studies (ATRANS) lab at Western New England University in Springfield, MA. Tablet-based training program. For the teen driver cohort, the study administrator had the participant interact with a practice drive on the tablet in order to let him or her get familiar with using tablet driving controls. Then participants were asked to interact with the eleven scenarios one by one. There was a latent hazard in each scenario. Participants were asked to drive with the iPad and tap on the hazardous area whenever they saw a hazard. If they tapped the correct area, the area became green. Then, for each scenario, a feedback video was played that described the potential hazards and what the driver should do to mitigate the mentioned hazards. At the end, if they were not successful in their first trial, the participants were asked to play the scenario again and try to mitigate the hazard. For the placebo teen driver cohort the administrator played a placebo training program for participants to make them believe they were undergoing training. Simulator Drive. Six months after training session, the teen participants in all cohorts were brought to the ATRANS lab and were asked to do a simulator based drive. The goal was to assess the tablet-based training effectiveness. In order to assess the level of the training effectiveness, participants put on an eye tracker and were asked to do the commentary driving with an emphasis on identifying potential hazards in the environment. Participants were seated in the driving simulator, then fitted and calibrated for the Tobii2 Mobile eye-tracker. Following a practice drive, participants were asked to drive three long drives, each 6 to 7 minutes. The drives were in different environments: commercial, neighborhood, and suburban. The participants' point-of-gaze were recorded continuously throughout the drive using the eye tracker. The participant's commentary driving was recorded as a part of the audio track in the eye tracker system.

Field Drive. Participants were scheduled for a field drive following the simulator drive. Participants wore the Tobii2 eye tracker and sat in the driving school's car. A driving instructor sat in front seat next to the participant. The instructor described what participants should do in terms of the hazard anticipation aspect of commentary driving. The instructor also provided navigation to the participants and reminded them to resume talking out loud if they kept silent during driving. The field drive took approximately twenty minutes for each participant. The drive included 22 scenarios based on what they learned in tablet-based training. The field drive scenarios are listed in Table 1.

Table 1. Hazard Scenarios and number of repetitions

\begin{tabular}{|c|l|c|}
\hline Item & Scenario Name & Quantity \\
\hline 1 & Right turns & 5 \\
\hline 2 & Crosswalks & 6 \\
\hline 3 & Obscured side roads on right & 1 \\
\hline
\end{tabular}

\begin{tabular}{|l|l|l|}
\hline 4 & Left turns & 5 \\
\hline 5 & Hidden driveways on right & 1 \\
\hline 6 & Controlled intersections & 3 \\
\hline 7 & Dangerous roundabout & 1 \\
\hline
\end{tabular}




\section{Analysis of Eye Tracking and Verbal Protocols}

In the case of assessing cognitive skills, verbal protocols provide insights into the information that a driver is processing and the meaning and priority they are applying to it. After collecting verbal reports, protocol analysis is used. Protocol analysis is an approach established to evaluate collected verbal reports. There are different methods defined by the possible outcomes of a task. A problem in various domains would have different types of solutions and answers. Therefore, different protocol analyses are required. After segmenting verbal protocols into text blocks, the participant's responses should be mapped to the possible or optimum solutions or a single current answer (Austin, J., \& Delaney, P. F. (1998)). In case of driving, to assess a driver's hazard anticipation ability according to Endsley's model, variables of interest are defined as follows for each level of Endsley's model: Level 1; to mention and fixate at any events or objects that require attention; Level 2; to explain about what is happening in the environment and gaze on an element two or three times; and Level 3; to project correctly what would be the future movements of objects in the environment.

Eye tracking protocols were also analyzed and mapped to Endsley's three level model utilizing blind review. Glancing at a cue was scored as perception (L1), while lingering for longer than 1 second or looking at a cue multiple times was scored as comprehension (L2). Glancing strategically toward areas that were previously obscured by the cues (such as the space in front of the bus at the crosswalk), was scored as projection (L3). In the Bus/Truck at a Midblock Crosswalk scenario, any fixation at the area in front of the bus indicated that participant perceived the hazard (L1). If the participant fixated for longer and/or scanned the hazardous area two or three times, the conclusion was that they achieved hazard comprehension (L2). Mitigation behavior with simultaneous longer fixation was the indication of projecting the hazard (L3).

\section{RESULTS}

In general, the results of the study showed that in comparison with control groups, trained groups mapped to a higher level of situation awareness. This difference happened in both simulator drive and field drive assessments. In the assessment of verbal protocol, teens in the placebo groups used discrete words, mostly including a list of what they saw at that particular moment rather than what they were in the process of doing. On the other hand, the verbal protocols for teens in the trained group verbal protocol included more detail. Their transcripts not only showed their ability to mention the potential hazards cues, but also indicated a higher order understanding of the potential for conflict in hazardous situations. This commonly came through as verbalizing what they should do to mitigate the potential hazards. The same happened in the eye tracking protocol. The trained group looked at the hazards more deliberately and gazed at hazardous areas more than once while their mitigating actions, like slowing down or pushing the brake pedal also proved their higher level of mental model.

The proportions in the Table 2 indicate the number of participants in each cohort who achieved L1, L2, and/or L3 situation awareness across all scenarios. It was generally assumed that if a participant achieved a higher level of situation awareness (say, L3), then they also achieved those levels below it. For instance, in the verbal protocol of the simulator drive in teen drivers, $63.49 \%$ of trained drivers achieved Level 1 SA compared with only $37.36 \%$ of placebos. $38.10 \%$ of trained reached Level 2 SA while only $20.51 \%$ of placebos did. Finally, $32.28 \%$ of trained drivers reached Level $3 \mathrm{SA}$ as compared to $15.75 \%$ of placebo drivers. Please note that the 
numbers do not add up to $100 \%$ because they do not reflect those drivers who failed to reach Level $1 \mathrm{SA}$ in a given scenario.

Eye tracking protocols and driver behaviors (slowing down, moving over, etc.) also seemed to follow this trend. The proportion results of the mapping of eye tracking protocols can also be found in Table 2. When considering eye tracking protocols and mapping them to SA, in simulator drive, trained teens achieved Level 3 SA 62.96 of the time whereas placebo group teens only achieved it $37.36 \%$ of the time.

Table 2. Proportional Analysis result

\begin{tabular}{|c|c|c|c|c|c|c|c|}
\hline & & \multicolumn{3}{|c|}{ Commentary } & \multicolumn{3}{|c|}{ Eye tracking } \\
\hline & Group & $\begin{array}{l}\text { L1 } \\
(\%)\end{array}$ & $\begin{array}{l}\text { L2 } \\
(\%) \\
\end{array}$ & L3 (\%) & $\begin{array}{l}\text { L1 } \\
(\%)\end{array}$ & $\begin{array}{l}\mathrm{L} 2 \\
(\%)\end{array}$ & $\begin{array}{l}\text { L3 } \\
(\%)\end{array}$ \\
\hline \multirow[b]{2}{*}{$\begin{array}{c}\text { Simulator } \\
\text { Drive }\end{array}$} & teen drivers & 63.49 & 38.10 & 32.28 & 74.60 & 62.96 & 62.96 \\
\hline & $\begin{array}{l}\text { placebo teen } \\
\text { drivers }\end{array}$ & 37.36 & 20.51 & 15.75 & 44.69 & 37.36 & 37.36 \\
\hline \multirow{2}{*}{$\begin{array}{l}\text { Field } \\
\text { Drive }\end{array}$} & teen drivers & 40.45 & 27.27 & 22.73 & 60.00 & 40.45 & 40.45 \\
\hline & $\begin{array}{l}\text { placebo teen } \\
\text { drivers }\end{array}$ & 24.79 & 15.70 & 11.16 & 49.17 & 24.79 & 24.79 \\
\hline
\end{tabular}

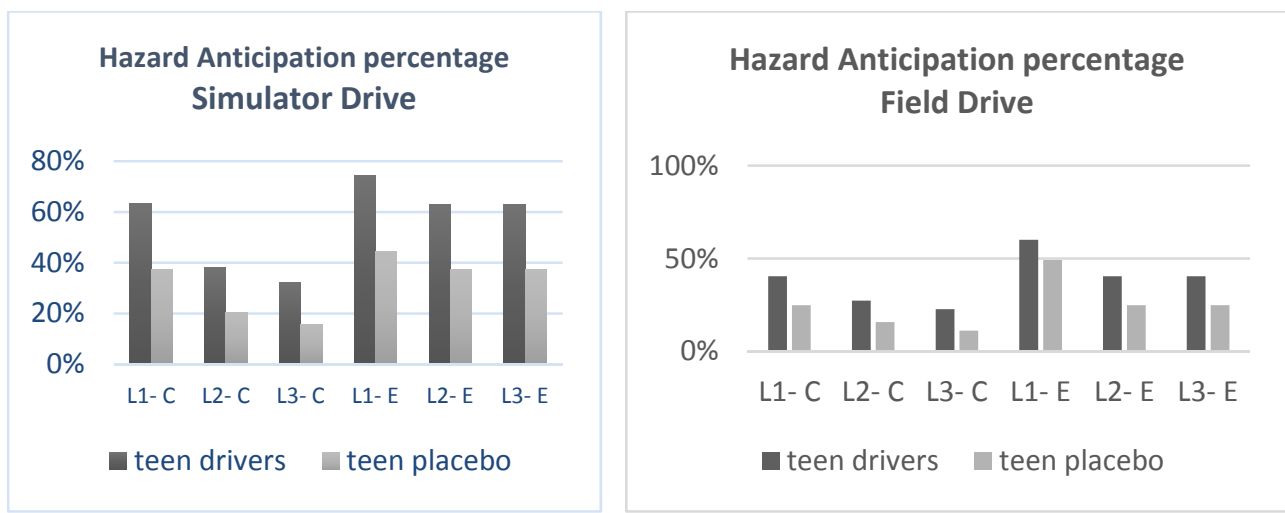

Figure 4. The Training Effectiveness

Using SPSS, the results show that there is a significant difference between teen drivers and placebo teen drivers cohort in achieving either levels of Endsley's model for commentary driving and the eye tracking, in both simulator and field drive (Table 3).

Table 3. Statistical Analysis result

\begin{tabular}{|c|l|c|c|}
\cline { 3 - 4 } \multicolumn{2}{c|}{} & Commentary & Eye tracking \\
\hline \multirow{3}{*}{ Simulator Drive } & Chi-square & 32.742 & 42.037 \\
\cline { 2 - 4 } & $p$-value & $<<0.001$ & $<<0.001$ \\
\cline { 2 - 4 } & $d f$ & 3 & 2 \\
\hline \multirow{3}{*}{ Field Drive } & Chi-square & 15.287 & 12.985 \\
\cline { 2 - 4 } & $p$-value & 0.002 & 0.002 \\
\cline { 2 - 4 } & $d f$ & 3 & 2 \\
\hline
\end{tabular}

\section{SUMMARY \& CONCLUSIONS}

Results demonstrated that the trained group reached a higher level of situation awareness. This occurred both in eye tracking and commentary driving. The primary goal of this study was to validate the effectiveness of an iPad training program. The results show significant differences 
between placebo and trained groups in processing environmental stimuli, mostly potential hazards. Trained groups were more able to describe what was going on in the environment and the impact of the hazardous cues. The mentioned the actual hazardous area more deliberately in comparison with placebo groups that labeled everything they saw in their environment as a hazard. However, a limitation of the study is that interpretation of the commentary road driving is difficult (Katrahmani, Romoser \& Samuel, 2016). In terms of eye tracking, the trained group both scanned and gazed at the hazardous area more tactically, while the placebo group seemed to randomly look at the hazards. The results also showed that in both sessions, teens were much better at recognizing and anticipating the hazards in simulator driving than in their field driving. This might be due to the fact that teens can identify and comprehend the hazards in theory. So, when they drive with the simulator, they act better than real life driving.

\section{ACKNOWLEDGEMENTS}

This research was supported by a grant from State Farm Insurance Company to Dr. Matthew Romoser. The authors would also like to thank their friends and colleagues Dr. Donald Fisher, Dr. Siby Samuel, Tracy Zafian, and all the student researchers at the University of Massachusetts Human Performance Laboratory for their continued collaboration and partnership in driver safety and training research.

\section{REFERENCES}

Braitman, K. A., Kirley, B. B., McCartt, A. T., \& Chaudhary, N. K. (2008). Crashes of novice teenage drivers: Characteristics and contributing factors. Journal of safety research, 39(1), 47-54.

Crundall, D. E., \& Underwood, G. (1998). Effects of experience and processing demands on visual information acquisition in drivers. Ergonomics, 41(4), 448-458.

Curry, A. E., Hafetz, J., Kallan, M. J., Winston, F. K., \& Durbin, D. R. (2011). Prevalence of teen driver errors leading to serious motor vehicle crashes. Accident Analysis \& Prevention, 43(4), 1285-1290.

DiMaggio, P. (1997). Culture and cognition. Annual review of sociology, 263-287.

Endsley, M. R. (1995). Toward a theory of situation awareness in dynamic systems. Human Factors: The Journal of the Human Factors and Ergonomics Society, 37(1), 32-64.

Goodwin, A., Foss, R., Margolis, L., \& Waller, M. (2010). Parents, teens and the learner stage of graduated driver licensing. Retrieved from http://trid.trb.org/view.aspx?id=968059

Hall, J., \& West, R. (1996). Role of formal instruction and informal practice in learning to drive. Ergonomics, 39(4), 693-706.

Katrahmani, A., Romoser, M., \& Samuel, S. (2016, September). Investigating a Non-Invasive Method of Measuring the Quality of Latent Hazard Schemas of Novice Teen and Experienced Adult Drivers A New Perspective using Traditional Tools. In Proceedings of the Human Factors and Ergonomics Society Annual Meeting (Vol. 60, No. 1, pp. 712-716). SAGE Publications.

Mayhew, D. R. (2007). Driver education and graduated licensing in North America: Past, present, and future. Journal of safety research, 38(2), 229-235.

McDonald, C. C., Curry, A. E., Kandadai, V., Sommers, M. S., \& Winston, F. K. (2014). Comparison of teen and adult driver crash scenarios in a nationally representative sample of serious crashes. Accident Analysis \& Prevention, 72, 302-308.

McKnight, A. J., \& McKnight, A. S. (2003). Young novice drivers: careless or clueless?. Accident Analysis \& Prevention, 35.6 (2003): 921-925.

Sagberg, F., \& Bjørnskau, T. (2006). Hazard perception and driving experience among novice drivers. Accident Analysis \& Prevention, 38(2), 407-414. 